\title{
The Diastolic Murmurs
}

\author{
Amar Narayan Patnaik ${ }^{1}$ \\ ${ }^{1}$ Department of Cardiology, Star Hospitals, Hyderabad, Telangana, \\ India
}

Ind J Car Dis Wom 2019;4:228-232

By definition, a murmur that starts with or after the second heart sound (S2) but ends at or before the first heart sound (S1) is called a diastolic murmur. Unlike the systolic murmurs which are quite prevalent even in those without a significant cardiac defect, the diastolic murmur almost all times suggests a significant cardiac abnormality.

\section{Scheme of Evaluation of a Murmur}

Any cardiac murmur including the diastolic murmurs should be assessed for the timing, intensity (grade), pitch (frequency), configuration, quality, location, and radiation. Sometimes maneuvers like inspiration/expiration, change of posture, leg rising, hand-grip, Valsalva/Muller, or use of amyl nitrate may have to be used to understand the true nature of a murmur. ${ }^{1}$

Timing: Diastolic murmur is more precisely timed as early (starts with A2 or P2 and ends at a variable distance in diastole but does not reach S1), mid (starts after A2 or P2 with a gap between the A2 or P2 and murmur, and end before S1), or late (starts well after the middle of the diastole but extends up to M1 or T1) (-Fig. 1). Duration: In general, the

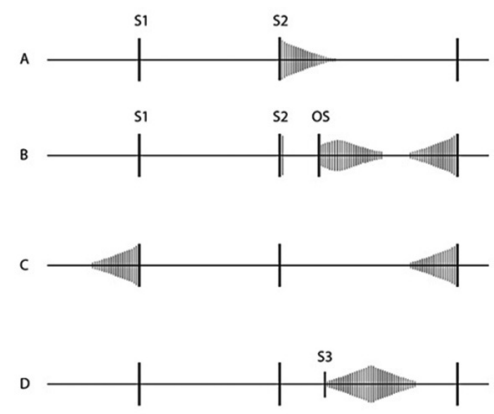

Fig. 1 The auscultogram pictures in various diastolic murmurs. (a) Early diastolic decrescendo murmur occupying less than $50 \%$ of diastole (mild chronic AR or PR). (b) Mid-diastolic murmur with presystolic murmur, that started with on opening snap-typical of a significant mitral stenosis. (c) Late diastolic murmur crescendo type. (d) Short mid-diastolic murmur after an S3 (generally a flow murmur). Abbreviations: S1, first heart sound; S2, second heart sound; S3, third heart sound; OS, opening snap.
Address for correspondence Amar Narayan Patnaik, MD, DM, FSCAI, FACC, Department of Cardiology, Star Hospitals, 8-2-594/B, Rd Number 10, Banjara Hills, Hyderabad, Telangana 500034, India (e-mail: anpatnaik@yahoo.com).

murmurs occupying more than $50 \%$ of diastole are regarded as long murmurs. Gradation of intensity: It can be graded into 6 as it is done conventionally for the systolic murmur, but some believe that using only 4 grades (grade I-barely audible, grade II-audible but soft, grade III-easily audible, and grade IV-loud and clear) is more appropriate. Frequency/ pitch/ configuration: The frequency (low, medium, and high), low (25-150 Hz) or high pitch and the configurations like crescendo or decrescendo are assessed. Low-pitched murmurs are best heard with bell of the stethoscope which is applied very light. High-pitched murmurs are best heard with the diaphragm of the stethoscope. These high-frequency murmurs can be missed in presence of noise in the doctor's clinic or the wards. With aging, the physicians may develop hearing difficulties with the high-pitched murmurs. Site of maximum intensity and transmission: This will give valuable information about the origin of a murmur.

Maneuvers and dynamic auscultation (- Table 1): An alteration in any of the features of a murmur due to change in posture, respiration, leg-raising, isometric hand grip, Valsalva/Muller's maneuvers, or by use of drugs like amyl nitrate may give a clue about the murmurs. Inspiration increases the murmur of mild to moderate pulmonic stenosis (PS), pulmonary regurgitation (PR), tricuspid regurgitation (TR), and tricuspid stenosis (TS). Expiration also augments mitral stenosis (MS) murmur. Valsalva-during strain phase, there is reduced venous return, decreased right and left ventricular volumes, and decreased systemic pressure leading to diminished intensity of flow murmurs as well as the murmurs of aortic regurgitation (AR), PR, and MS. Amyl nitrate inhalation causes potent systemic vasodilatation resulting in decreasing the intensity of AR murmur. It is followed by reflex tachycardia which increases the right-sided murmurs. ${ }^{1}$

The diastolic murmurs are classified as early, mid, or late diastolic murmurs based on the timing and they can be caused by a variety of clinical entities as outlined in - Table 2 .

\section{Early Diastolic Murmurs}

Semilunar valve damage leads high velocity regurgitant flow in early diastole from the great artery into the
DOI https://doi.org/ $10.1055 / \mathrm{s}-0039-3402692$
(C)2019 Women in Cardiology and Related Sciences
License terms

()(1) $\Theta \circledast$ 
Table 1 Dynamic auscultation to define the diastolic murmurs

\begin{tabular}{|c|c|c|c|c|c|c|}
\hline & Change in posture & Inspiration/expiration & Leg rising & Hand-grip & Valsalva-strain & Amy Initrate \\
\hline MS & Left lateral & $\begin{array}{l}\text { Expiration augments MS } \\
\text { murmur }\end{array}$ & & $\begin{array}{l}\text { Murmur } \\
\text { increases }\end{array}$ & $\begin{array}{l}\text { Diminished } \\
\text { intensity }\end{array}$ & Murmur increases \\
\hline TS & Leg raising & $\begin{array}{l}\text { Inspiration increases the } \\
\text { murmur of TS }\end{array}$ & $\begin{array}{l}\text { Increased } \\
\text { murmur }\end{array}$ & & & Murmur increases \\
\hline AR & Sitting, leaning forward & Held expiration & & $\begin{array}{l}\text { Murmur } \\
\text { increases }\end{array}$ & $\begin{array}{l}\text { Increased } \\
\text { intensity }\end{array}$ & Diminished intensity \\
\hline PR & & $\begin{array}{l}\text { Inspiration increases the } \\
\text { murmur of PR }\end{array}$ & $\begin{array}{l}\text { Increased } \\
\text { murmur }\end{array}$ & & & Murmur increases \\
\hline
\end{tabular}

Table 2 Conditions producing diastolic murmurs

\begin{tabular}{|c|c|c|}
\hline & Common & Uncommon \\
\hline EDM & $\begin{array}{l}\text { 1. Chronic aortic regurgitation (congenital valve deformity, rheumatic, endocarditis, } \\
\text { prolapse, postsurgical or balloon Valvotomy, trauma, dilated aortic root/annulus, aortic } \\
\text { dissection). } \\
\text { 2. Pulmonary regurgitation (secondary to PHTN, congenital defect, postvalvuloplasty, } \\
\text { post-ICR for TOF, endocarditis, annular dilation due to Marfan/other collagen disorders } \\
\text { or idiopathic dilatation of PA) }\end{array}$ & $\begin{array}{l}\text { Key Hodgkin's murmur } \\
\text { Cole Cecil murmur } \\
\text { Dock's murmur } \\
\text { Cabot Locke murmur } \\
\text { Carcinoid disease } \\
\text { Rheumatic PR } \\
\text { Catheter-induced trauma to PV }\end{array}$ \\
\hline MDM & $\begin{array}{l}\text { 1. Mitral/tricuspid stenosis } \\
\text { 2. Carey Coombs murmur } \\
\text { 3. LA/RA myxoma } \\
\text { 4. Carcinoid } \\
\text { 5. Flow murmurs in ASD, PDA, VSD, or severe MR/TR } \\
\text { 6. Austin Flint murmur }\end{array}$ & Prosthetic valve \\
\hline LDM & Presystolic murmurs (MS/TS) & $\begin{array}{l}\text { Rytand's murmur: } \\
\text { In CHB }\end{array}$ \\
\hline
\end{tabular}

Abbreviations: CHB, complete hear block; PA, pulmonary artery; PHTN, pulmonary hypertension.

respective ventricle. This creates a high-pitched murmur starting with the semilunar valve closure. Decrescendo configuration of the murmur is typical representing the pressure change. Generally large regurgitant volume makes the murmur longer, but the correlation is wellmatched. Inspiration increases the pulmonary regurgitant murmur and at held expiration the AR murmur is best heard. Chronic aortic regurgitation and pulmonary regurgitation are the most common causes of an end diastolic murmur (EDM).

1. Chronic aortic regurgitation. High-pitched blowing decrescendo murmur is typical character of severe chronic AR. The common entities that cause aortic regurgitation include congenital valve deformity, rheumatic, endocarditis, prolapse, postsurgical or balloon valvotomy, trauma, dilated aortic root/annulus, aortic dissection, and so forth. Isometric handgrip increases systematic vascular resistance (SVR) and AR murmur increases. The murmur may have a musical quality (diastolic whoop), when it occurs due to everted aortic cusp. The murmurs due to primary aortic valve disease are best heard at left parasternal areas, whereas those due to dilated aortic root are often well heard at the right sternal border. Sitting/leaning forward posture will make the AR murmur more audible. Held expiration is the best time to hear the AR murmur clearly. It can be augmented by maneuvers that increase afterload like squatting and on isometric handgrip. Additionally, a systolic murmur due to the large stroke volume and Austin Flint murmur may be heard.

2. Acute AR: The murmur is softer and shorter because the LV diastolic pressure rises rapidly diminishing the aortic-LV pressure gradient. Additionally, tachycardia, soft S1, and absence of signs of aortic runoff are the clue to diagnose acute/subacute AR. The EDM is softer and shorter in acute AR. It can have a medium frequency.

EDM due to pulmonary regurgitation can occur due to a variety of conditions like pulmonary hypertension (Graham Steell murmur), congenital defect, postvalvuloplasty, postICR for TOF, endocarditis, carcinoid disease, annular dilation due to Marfan/other collagen disorders, or idiopathic dilatation of PA. Other rare causes of low-pressure PR include carcinoid syndrome, rheumatic valve disease, and catheter induced trauma.

3. PR associated secondary to PAH: It produces a variable duration of localized (best heard in left second and third intercostal spaces) high-pitched blowing early decrescendo diastolic murmur like AR murmur which increases with inspiration. It is the most common example of PR and is popularly known as Graham Steell murmur. ${ }^{2}$

4. PR that occurs following repair of tetralogy of fallot (TOF) is typically low-pitched and soft due to normal PA pressures. There may be a to-and-fro murmur or no murmur at all. - Table 3 highlights important differences between AR and PR murmur. 
Table 3 Differentiating AR murmur from PR murmur

\begin{tabular}{|l|l|l|}
\hline Feature & AR murmur & PR murmur \\
\hline Best site & Aortic area & Pulmonary area \\
\hline Posture & Sitting, leaning forward & Supine \\
\hline Signs of run off & present & Absent \\
\hline Apex & $\begin{array}{l}\text { LV type (hyperdynamic } \\
\text { and unsustained) }\end{array}$ & RV type \\
\hline $\begin{array}{l}\text { Associated } \\
\text { signs }\end{array}$ & - & Loud P2, RV lift, MS \\
\hline
\end{tabular}

\section{Mid-Diastolic Murmurs}

Mitral stenosis produces the prototype mid-diastolic murmur (MDM). A narrowed mitral valve produces a pressure gradient between the left atrium and the left ventricle. The gradient is increased by increase in heart rate or cardiac output. This murmur is generated due to increased blood flow through the narrowed mitral valve in presence of a diastolic pressure gradient across the valve. Besides mitral stenosis, tricuspid stenosis, Carey Coombs murmur, LA myxoma, flow murmurs in ASD, PDA, VSD, or severe MR/TR can all cause a $\operatorname{MDM}$ (- Table 1).

1. Mitral stenosis: The classical murmur is described as low-pitched rough rumbling MDM with presystolic accentuation best heard at the apex, especially in left lateral position with bell of the stethoscope. Openings snap if present, precedes the murmur onset. In late diastole the atrial contraction further increases the pressure gradient producing the typical presystolic murmur. The duration of MDM correlates with the severity of mitral stenosis and is useful as a useful clinical clue. Loudness of the MDM is not consistently related to severity of MS. In severe mitral stenosis when associated with severe right heart failure and pulmonary hypertension the duration of the murmur may be shortened. In high output states like pregnancy the duration of murmur may be misleadingly longer. Similarly, during atrial fibrillation, the longer cycles will have longer duration of MDM and shorter cycles may have very brief diastolic rumble. In very obese or if the cardiac output is less or due to extreme rotation of heart the murmur may not be heard (silent MS). However, it is important to note that loudness does not reflect the severity. Additionally loud $\mathrm{S} 1$ and OS favor the diagnosis of MS.

2. Carey Coombs murmur: In acute rheumatic fever due to acute valvulitis (causing edema of leaflets) an MDM may be heard at the apex. Some believe first degree heart-block so frequent in acute carditis may cause early atrial systole and the resultant increased flow may coincide with rapid filling phase and causes this murmur.

3. LA myxoma: LA myxoma can produce a similar murmur and can be confused with MS. It is frequently presystolic in timing and crescendo in configuration. Changing intensity and character of the murmur with alteration in posture is typical of LA myxoma. The murmur begins when the tumor is pushed toward the mitral valve with onset of ventricular systole while the flow across the valve is continuing. Most myxomas do not produce any murmur. A tumor-plop, a low-pitched murmur may be heard in a few cases especially in some postures.

4. Tricuspid stenosis: Rheumatic tricuspid valve involvement almost always occurs along with mitral valve lesions. The TS murmur is scratchy and becomes longer and louder by any maneuver that increases the venous return like inspiration, leg rising, exercise, or Muller's maneuver. The two murmurs can be distinguished separately based on the character and location (-Table 4 ). The murmur resembles mitral stenotic murmur but increases in intensity on inspiration (Carvallo's sign) and may be confined to late diastole. There may be an opening snap and an occasional split first heart sound. The a-wave in jugular venous pressure (JVP) may be prominent. In presence of atrial fibrillation, the TS murmur may be more obscured causing considerable difficulty. Carcinoid valve disease or right atrial myxoma can also cause isolated tricuspid valve disease.

5. Austin Flint murmur: In severe AR, sometimes a midlate low-pitched diastolic murmur may be heard at apex, which can be distinguished from murmur of MS based on associated auscultatory findings and the response of the murmur to vasodilators ( $\mathbf{- T a b l e ~ 5 )}$ ). Decrease in afterload decreases the severity of AR and the intensity of Austin Flint murmur. The murmur is generated due to the jet of aortic regurgitation causing fluttering or due to functional mitral stenosis. Some believe the AR jets directed to the LV free wall can also cause this murmur. ${ }^{3}$

6. Prosthetic valves: In a patient with normal mitral prosthetic valve the closing sounds (high-frequency with metallic tone) are well heard even without a stethoscope. Bioprosthetic valve and rarely St. Jude valve may cause a short diastolic murmur at apex in left lateral decubitus

Table 4 Comparison of TS murmur with MS murmur

\begin{tabular}{|l|l|l|}
\hline Feature & $\begin{array}{l}\text { Tricuspid stenosis } \\
\text { murmur }\end{array}$ & $\begin{array}{l}\text { Mitral stenosis } \\
\text { murmur }\end{array}$ \\
\hline Best heard & Left lower sternal border & $\begin{array}{l}\text { Apex (left lateral } \\
\text { decubitus) }\end{array}$ \\
\hline Timing & $\begin{array}{l}\text { Starts early in diastole } \\
\text { with opening snap; does } \\
\text { not increase up to S1 }\end{array}$ & $\begin{array}{l}\text { Increases through the } \\
\text { diastole }\end{array}$ \\
\hline Duration & Short & Long \\
\hline Character & Scratchy & $\begin{array}{l}\text { Rumbling- } \\
\text { low-pitched }\end{array}$ \\
\hline Increases by & Inspiration & Exercise \\
\hline
\end{tabular}

Table 5 Differentiation of MS from Austin Flint due to severe AR

\begin{tabular}{|l|l|l|}
\hline Feature & Mitral stenosis murmur & Austin Flint murmur \\
\hline Rhythm & Atrial fibrillation frequent & Generally sinus rhythm \\
\hline Apex & Tapping & LV heave \\
\hline RV lift & Present & Absent \\
\hline S1 & Present & Absent \\
\hline OS & Common & Absent \\
\hline S3 & Absent & $+/-$ \\
\hline
\end{tabular}


position. A degenerating bioprosthetic valve or a slowly thrombosing mechanical valve may present with a MDM mimicking native valve stenosis. At aortic position, even normally functioning tilting disc or bileaflet valve may have some low-intensity short diastolic murmur. For a cage-balled prosthesis any diastolic murmur is considered abnormal.

7. Flow murmurs due to a large left-to-right shunt: The flow murmurs can be of low to medium (120-330 cps) frequency. These unimpressive MDMs dependent on magnitude of the shunt across and the resultant increase in flow across the respective AV valves. In ASD, the flow murmur is best heard at lower left sternal border and increases in inspiration. Murmur is variable, sometimes scratchy, and may increase on inspiration or leg elevation. If RV is too large it may be heard at the apex. In PDA and VSD a similar diastolic flow murmur may be heard across the mitral valve. These flow murmurs are short in duration and usually proceed by a loud $\mathrm{S} 3$. There is no OS or presystolic murmur associated.

8. Flow murmur in severe mitral regurgitation: In pre severe mitral regurgitation with no stenosis large volume of blood that moves from left atrium to left ventricle in diastole can result in a situation of functional mitral stenosis, as the mitral valve is wide open at the beginning of diastole and it partially closes with the continued flow.

\section{Late Diastolic Murmurs}

The best example is the presystolic component of the mitral stenotic murmur, which is thought to be due to atrial contraction that increases the ventricular filling in late diastole. This murmur is thought to occur only in sinus rhythm as the atrial contraction is a prerequisite. But later some authors observed that such murmurs can occur even in atrial fibrillation. It is due to a persistent diastolic gradient during short cycles, reflecting continuous diastolic flow across the mitral valve. A continued narrowing of mitral valve late in diastole produces a presystolic murmur.

\section{Effect of an Associated Lesion on Murmur that Is Being Assessed}

In clinical practice, combination of valve lesions is not uncommon. The presence of a lesion in the other valve of a combination of stenosis with regurgitation at the same valve can present a change in the murmur that is being evaluated.

- MS+MR: Because MR itself can cause a mid-diastolic rumble one should concentrate on length of the murmur. A long MDM with a presystolic component favors dominant MS. Length of murmur rather than the intensity will suggest the severity of mitral stenosis. Additionally, loud first sound, opening snap, prominent presence of atrial fibrillation, and impressive parasternal lift favor stenosis as the dominant lesion.

- $\boldsymbol{A S + A R : ~ S i g n i f i c a n t ~ A S ~ c a n ~ m a k e ~ t h e ~ A R ~ m u r m u r ~ s h o r t ~ a s ~}$ well as decapitate the systolic pressure and mask the signs of aortic runoff. Significant AR gives a hyperdynamic and ill-sustained apex.

- MS+AR: Presence of MS may attenuate the AR murmur and the LV dilatation is also lesser.

- MS+ASD: In situations where LA pressure is raised and there is a small ASD, a continuous or a diastolic murmur may be generated. ${ }^{\mathrm{Q} 4}$ Such murmur can be scratchy or crackling audible at left second or third intercostal space, may radiate to apex or right of sternum. It can be of varying duration and is augmented on inspiration

Named Diastolic Murmurs: Various named diastolic murmurs are described which are often confusing to the students; yet, they form fancy questions in viva/bed-side examination (-Table 6). Austin Flint murmur, Carey Coombs murmur, and Graham Steell murmur are described already (see the earlier sections).

Rytand's murmur: In complete heart block (CHB), when ventricular rate is very slow, there can be a diastolic mitral regurgitation resulting in a late short diastolic murmur. ${ }^{4}$

Dock's murmur: Dock's murmur is early diastolic and decrescendo sounding like the murmur of aortic regurgitation that

Table 6 Examples of named diastolic murmurs

\begin{tabular}{|l|l|l|}
\hline Named murmur & Characteristics & Remarks \\
\hline Austin Flint murmur & Mid-late low-pitched diastolic murmur at apex & In severe AR: differentiate from MS murmur \\
\hline Carey Coombs murmur & Mid-diastolic murmur at apex & In acute rheumatic fever due to acute valvulitis \\
\hline Graham Steell murmur & High-pitched blowing early decrescendo diastolic murmur & Severe pulmonary hypertension \\
\hline Rytand's murmur & Late short diastolic murmur & In CHB, a diastolic mitral regurgitation \\
\hline Dock's murmur & Early diastolic and decrescendo murmur & Severe stenosis of LAD \\
\hline Cabot Locke Murmur & Early diastolic and decrescendo murmur & $\begin{array}{l}\text { High flow through LMCA or LAD in anemic } \\
\text { patients }\end{array}$ \\
\hline Cole Cecil murmur: & The early diastolic murmur of AR is heard best in the axilla & Attributed to high placed apex \\
\hline Key Hodgkin's murmur & $\begin{array}{l}\text { Early diastolic and decrescendo rasping murmur sounds } \\
\text { like saw cutting through wood }\end{array}$ & $\begin{array}{l}\text { Due to retroversion of aortic leaflets seen in } \\
\text { syphilitic valve disease }\end{array}$ \\
\hline
\end{tabular}

Abbreviations: LAD, left anterior descending artery; LMCA, left main coronary artery. 
can be heard when there is severe stenosis of the left anterior descending coronary artery. Other signs of aortic run off do not occur along with this murmur. ${ }^{5}$

Cabot-Locke murmur: High flow through LMCA or LAD in anemic patients can cause this early diastolic murmur.

Cole-Cecil murmur: When the early diastolic murmur of AR is heard best in the axilla it is given this name. It is attributed to high placed apex. ${ }^{6}$

Key-Hodgkin's murmur: EDM of aortic regurgitation due to retroversion of aortic leaflets seen in syphilitic valve disease. The murmur resembles the sound of saw cutting through wood with rasping quality.

\section{Conclusion}

Knowing the various aspects of the diastolic murmur and its interpretation in the light of associated cardiac findings and the clinical background makes the clinical evaluation a very satisfying experience.

\section{Conflict of Interest}

None.

\section{References}

1 Abrams J. Essentials of Cardiac Physical Diagnosis. Philadelphia, PA: Lea \& Febiger; 1987

2 Cohn KE, Hultgren HN. The Graham-Steell murmur re-evaluated. N Engl J Med 1966;274(9):486-489

3 Foth C, Zeltser R. Austin Flint Murmur. [Updated 2018 Dec 16]. In: Stat Pearls [Internet]. Treasure Island (FL): Stat Pearls Publishing; 2019

4 Panidis IP, Ross J, Munley B, Nestico P, Mintz GS. Diastolic mitral regurgitation in patients with atrioventricular conduction abnormalities: a common finding by Doppler echocardiography. J Am Coll Cardiol 1986;7(4):768-774

5 Sangster JF, Oakley CM. Diastolic murmur of coronary artery stenosis. Br Heart J 1973;35(8):840-844

6 Cole R, Cecil AB. The axillary diastolic murmur in aortic insufficiency. Bull Johns Hopkins Hosp 1908;19:353 